Jack R. L. McLean

David P. Inwald

\section{The utility of stroke volume variability as a predictor of fluid responsiveness in critically ill children: a pilot study}

Accepted: 20 November 2013

Published online: 5 December 2013 (C) The Author(s) 2013. This article is published with open access at Springerlink.com

Dear Editor,

Predicting the response to fluid bolus therapy in critically ill children is difficult [1]. However, rapid bolus fluid resuscitation is integral to the management of children presenting with severe sepsis [2]. Bolus was compared with maintenance fluid in a relatively recent large randomized controlled trial carried out in in the developing world (the Fluid Expansion As Supportive TherapyFEAST) that involved a large cohort of African children with severe infection [3]. The surprising result of FEAST was that bolus fluid was associated with a $30 \%$ increase in mortality.

Methods to predict fluid responsiveness in children requiring paediatric intensive care unit (PICU) admission might therefore be extremely useful. Previous paediatric studies have highlighted the utility of Doppler-based methods [4] and have demonstrated that stroke volume variability (SVV) predicts fluid responsiveness [5]. However, data in a PICU setting are currently limited. The aim of our pilot study was to evaluate the utility of SVV as a predictor of fluid responsiveness in a PICU.

We studied a group of 13 mechanically ventilated children, aged 2 months to 14 years, who were given fluid boluses in the PICU at $\mathrm{St}$ Mary's Hospital, London, from 18 March 2013 to 13 May 2013. Exclusion criteria were high-frequency oscillatory ventilation, arrhythmia and left to right shunt. The study design was reviewed and approved by Brent Research Ethics Committee. Written informed consent was obtained from an individual with parental responsibility.

Heart rate and blood pressure were continuously monitored on an Intellivue MP70 patient monitor (Philips Healthcare, Guildford, UK). The data collected on these parameters were subsequently analysed as the mean of three readings each timed $1 \mathrm{~min}$ apart. Stroke volume index (SVI) and SVV were measured non-invasively at the aortic valve using suprasternal Doppler (USCOM Ltd., Sydney, Australia). SVV is calculated automatically as: $\left(\mathrm{SV}_{\max }-\mathrm{SV}_{\min }\right) /$ $\left[\left(\mathrm{SV}_{\max }+\mathrm{SV}_{\min }\right)\right] \times 100$ from a 7 -s data window. Doppler traces were

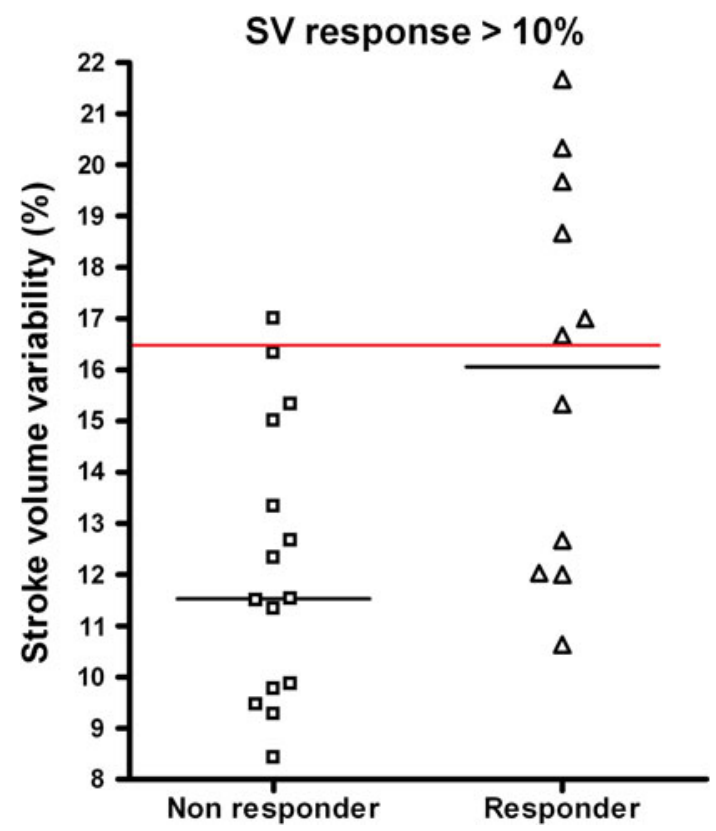

Fig. 1 Stroke volume variability (SVV) in responsive (increase in SVI of $>10 \%$ ) and nonresponsive patients following administration of fluid bolus. Black horizontal lines indicate median values for each group, red horizontal line indicates the $16.5 \%$ cutoff identified recorded in triplicate, and the values of SVI and SVV used in the analysis were the mean of three measurements.

After the initial data collection immediately prior to the administration of fluid bolus, $10 \mathrm{ml} / \mathrm{kg}$ fluid bolus was administered. Data were collected again immediately post fluid bolus. Patients were defined as either responsive or non-responsive to each individual fluid bolus, with responsive defined as an increase in SVI of at least $10 \%$.

Data on a total of 26 fluid boluses were analysed. In 11 boluses, patients were classified as responsive and in 15 non-responsive. Static haemodynamic parameters did not differ significantly between the two groups, and ventilation and inotrope use was unchanged.

SVV differed significantly between the responsive (median $16.67 \%$, range $12.35-19.17 \%$ ) and nonresponsive groups $(11.53 \%$, range 9.82-14.17\%) $(p=0.01)$ (Fig. 1). 
The receiver operating characteristic curve analysis for pre-bolus SVV to predict a $>10 \%$ rise in SVI postbolus found an area under the curve of 0.797 (95\% confidence interval $0.623-0.971, p=0.01)$. The Youden index calculations identified an optimal cutoff for SVV of $16.5 \%$ (sensitivity $54.5 \%$, specificity $93.3 \%$ ). This cutoff has a positive likelihood ratio of 8.18 and a negative likelihood ratio of 0.49 .

Taken together, these results suggest that SVV has the potential to predict fluid responsiveness in mechanically ventilated children in the PICU. The small sample size of our pilot study limits generalizability, and further work is required to confirm and extend these findings.

Acknowledgments Dr. Inwald is grateful for support from HEFCE and the NIHR
Biomedical Research Centre funding scheme.

\section{Conflicts of interest None.}

Open Access This article is distributed under the terms of the Creative Commons Attribution Noncommercial License which permits any noncommercial use, distribution, and reproduction in any medium, provided the original author(s) and the source are credited.

\section{References}

1. Tibby SM, Hatherill M, Marsh MJ et al (1997) Clinicians' abilities to estimate cardiac index in ventilated children and infants. Arch Dis Child 77:516-518

2. Brierley J, Carcillo JA, Choong K et al (2009) Clinical practice parameters for hemodynamic support of pediatric and neonatal septic shock: 2007 update from the American College of Critical Care Medicine. Crit Care Med 37:666-688
3. Maitland K, Kiguli S, Opoka RO et al (2011) Mortality after fluid bolus in African children with severe infection. N Engl J Med 364:2483-2495

4. Durand P, Chevret L, Essouri S et al (2008) Respiratory variations in aortic blood flow predict fluid responsiveness in ventilated children. Intensive Care Med 34:888-894

5. Renner J, Broch O, Duetschke P et al (2012) Prediction of fluid responsiveness in infants and neonates undergoing congenital heart surgery. Br J Anaesthesia 108:108-115

\section{J. R. L. McLean (匹)}

Faculty of Medicine, Imperial College

London, South Kensington Campus,

London SW7 2AZ, UK

e-mail: jack.mclean09@imperial.ac.uk

Tel.: +44-07503599521

D. P. Inwald

Paediatric Intensive Care Unit, St Mary's

Hospital, Imperial College Healthcare NHS

Trust, London W2 1NY, UK 